\title{
Perfil de pacientes em hemodiálise de um serviço de referência do interior do Rio
}

\section{Grande do Sul, Brasil}

\author{
Patient profile in hemodialysis from a referral service in Rio Grande do Sul, Brazill \\ Perfil de paciente en hemodiálisis de un servicio de referencia en interior de Rio Grande do Sul,
}

Brasil

Recebido: 21/12/2021 | Revisado: 28/12/2021 | Aceito: 07/01/2022 | Publicado: 10/01/2022

Marri Andressa Hilbert de Souza
ORCID: https://orcid.org/0000-0003-2074-8043
Hospital Bom Jesus, Brasil
E-mail: marrisouza@ sou.faccat.br
Rubellita Holanda
ORCID: https://orcid.org/0000-0002-3690-9991
Faculdades Integradas de Taquara, Brasil
E-mail: rubellitaholanda @ faccat.br
Edna Thaís Jeremias Martins
ORCID: https://orcid.org/0000-0002-2546-2987
Faculdades Integradas de Taquara, Brasil
E-mail: ednamartins@ faccat.br
Andriele Cristina Hoffmeister
ORCID: https://orcid.org/0000-0003-4357-1471
Faculdades Integradas de Taquara, Brasil
E-mail: andriele.hoffmeister@ sou.faccat.br
Claudia Capellari
ORCID: https://orcid.org/0000-0002-2003-1424
Faculdades Integradas de Taquara, Brasil
E-mail: claudiacapellari @ faccat.br

\section{Resumo}

Objetivo: Identificar o perfil sociodemográfico e clínico de pacientes com Doença Renal Crônica em hemodiálise em um serviço de referência no interior do Rio Grande do Sul, RS. Metodologia: Estudo quantitativo, transversal e com característica de censo. Participaram 94 pacientes com Doença Renal Crônica (DRC), em tratamento hemodialítico, vinculados a um serviço de nefrologia do interior do Rio Grande do Sul, Brasil. A coleta de dados ocorreu entre setembro e outubro de 2020, por meio de consulta de enfermagem, exames de rotina, prontuários e questionários estruturados. Resultados: 94 pacientes foram incluídos no estudo, com idade média de $53,1( \pm 13,7)$ anos, prevalência do sexo masculino (61,7\%), casados $(45,7 \%)$, renda de 1 a 2 salários mínimos (46,8\%), escolaridade com fundamental incompleto $(62,8 \%)$, cor branca $(80,9 \%)$. Quanto aos aspectos clínicos, a principal etiologia da DRC foi a hipertensão $(43,6 \%)$, o tempo de diagnóstico foi em média $6,05( \pm 6,46)$ anos, acesso venoso por fístula arteriovenosa para hemodiálise $(94,7 \%)$. As principais complicações foram hipotensão $(56,4 \%)$ e câimbras $(28,7 \%)$. Conclusão: Na amostra, prevaleceram homens brancos, casados e de baixa escolaridade, com hipertensão arterial como principal fator etiológico. A variabilidade dos achados aponta para a necessidade de individualização do cuidado a essa população.

Palavras-chave: Diálise renal; Estudos transversais; Doença renal crônica.

\begin{abstract}
Objective: To identify the sociodemographic and clinical profile of patients with Chronic Kidney Disease undergoing hemodialysis in a reference service. Methodology: Quantitative, cross-sectional study with characteristics of a census. 94 patients with Chronic Kidney Disease (CKD) undergoing hemodialysis, linked to a nephrology service participated in this study. Data collection took place between September and October 2020, through nursing consultations, routine exams, medical records and structured questionnaires. Results: 94 patients were included in the study, with a mean age of 53.1 ( \pm 13.7 ) years, prevalence of males $(61.7 \%)$, married $(45.7 \%)$, income from 1 to 2 minimum wages $(46.8 \%)$, with incomplete primary education $(62.8 \%)$, white $(80.9 \%)$. As for clinical aspects, the main etiology of CKD was hypertension $(43.6 \%)$, time since diagnosis on average $6.05( \pm 6.46)$ years, venous access by arteriovenous fistula for hemodialysis $(94.7 \%)$. The main complications were hypotension $(56.4 \%)$ and cramps $(28.7 \%)$. Conclusion: In the sample, was prevalent white men, married and with low education prevailed, with arterial hypertension being the main etiological factor. The variability of the findings points to the need for individualization of care for this population.
\end{abstract}

Keywords: Renal dialysis; Cross-sectional studies; Chronic renal insufficiency. 


\begin{abstract}
Resumen
Objetivo: Identificar el perfil sociodemográfico y clínico de pacientes con Enfermedad Renal Crónica en hemodiálisis en un servicio de referencia en el interior de Rio Grande do Sul, RS. Metodología: Estudio cuantitativo, transversal con características de censo. En este estudio participaron 94 pacientes con Enfermedad Renal Crónica (ERC) en hemodiálisis, vinculados a un servicio de nefrología en el Rio Grande do Sul, Brasil. La recolección de datos se realizó entre septiembre y octubre de 2020, a través de consultas de enfermería, exámenes de rutina, historias clínicas y cuestionarios estructurados. Resultados: Se incluyeron en el estudio 94 pacientes, con una edad media de 53,1 ( $\pm 13,7$ ) años, prevalencia de varones $(61,7 \%)$, casados (45,7\%), ingresos de 1 a 2 salarios mínimos (46,8\%), educación con educación primaria incompleta $(62,8 \%)$, blancos $(80,9 \%)$. En cuanto a los aspectos clínicos, la principal etiología de la ERC fue la hipertensión arterial $(43,6 \%)$, tiempo desde el diagnóstico en promedio 6,05 $( \pm 6,46)$ años, acceso venoso por fístula arteriovenosa para hemodiálisis (94,7\%). Las principales complicaciones fueron hipotensión (56,4\%) y calambres (28,7\%). Conclusión: En la muestra predominaron los hombres blancos, casados y con bajo nivel educativo, siendo la hipertensión arterial el principal factor etiológico. La variabilidad de los hallazgos apunta a la necesidad de individualizar la atención para esta población.
\end{abstract}

Palabras clave: Diálisis renal; Estudios transversales; Insuficiencia renal crónica.

\title{
1. Introdução
}

A Doença Renal Crônica (DRC) é considerada um problema de saúde pública crescente em todo o mundo e está associada a custos significativos aos sistemas de saúde (Vallianou, et al, 2019). A DRC afeta de 8\% a 16\% da população mundial, sendo uma das principais causas de morte e também a $16^{\text {a }}$ causa principal de anos de vida perdidos em todo o mundo. O diagnóstico e manejo adequado são importantes na prevenção de complicações associadas a essa patologia (Chen, et al., 2019). A DRC é definida como a perda funcional e estrutural dos rins, presentes por mais de 3 meses, que geram implicações para a saúde (KDIGO, 2017)

No Brasil, observa-se um aumento progressivo do número de pacientes prevalentes em diálise. O último inquérito estimou que 139.691 pessoas realizavam diálise crônica no país no ano 2019, sendo que 93,2\% das mesmas utilizavam a hemodiálise como modalidade de tratamento (Neves, 2021). Em outros países, como Estados Unidos, a taxa de pacientes em hemodiálise é de 89,9\% (USRDS, 2019) e, na Europa, é de 85\% (Kramer et al., 2020). A nível regional brasileiro, o Sul e o Centro-Oeste têm as maiores taxas anuais de incidência de pacientes dialíticos, sendo a maior taxa global da América Latina (Neves et al., 2021; Neves, 2020). Contudo, observa-se que a população de pacientes em diálise continua a crescer rapidamente, possivelmente, como resultado de um aumento da expectativa de vida da população (Zheng et al.,2020), aumento da prevalência de hipertensão e diabetes mellitus e exposições ambientais tóxicas.

É por meio da diálise que ocorre a remoção de toxinas, onde esses solutos atravessam a membrana semipermeável seguindo um gradiente de concentrações. A dinâmica da terapia de substituição renal varia entre países com a definição de tempo após avaliação do nível de lesão renal (Murdeshwar \& Anjum, 2021). O procedimento comumente é realizado de três a quatro vezes por semana, por um tempo médio de quatro horas.

Atualmente o Sistema Único de Saúde (SUS) é considerado como a principal fonte pagadora do tratamento hemodialítico no Brasil (79\%) (Neves et al., 2021), mesmo que as clínicas privadas sejam predominantes, representando um número de 70 a $72 \%$ (Neves, et. al., 2020).

A partir dos resultados apresentados neste estudo, será possível conhecer a realidade dos pacientes em hemodiálise, que contribuirá para a melhoria da qualidade de cuidado prestado ao paciente em hemodiálise. Com isso, contribuir com a produção científica em enfermagem, tendo em vista a carência de pesquisas e artigos publicados no Brasil nos últimos 2 anos.

O objetivo deste trabalho foi identificar o perfil sociodemográfico e clínico de pacientes com Doença Renal Crônica em hemodiálise em um serviço de referência no interior do Rio Grande do Sul, Brasil. 


\section{Metodologia}

Trata-se de um estudo descritivo transversal, de abordagem quantitativa, com amostra do tipo censo, quando são incluídos todos os casos do universo de estudo da população (Sampieri et al.,2013). A coleta de dados foi realizada no serviço de nefrologia do município de Taquara, Rio Grande do Sul (RS), Brasil, de setembro a outubro de 2020.

Como critérios de inclusão foram pacientes em tratamento hemodialítico, vinculados ao serviço de diálise em questão, maiores de 18 anos. Como critérios de exclusão, considerou-se tratamento intensivo, recuperação da função renal, transferência para outro serviço de diálise, a transição para outro método dialítico, transplante renal ou óbito.

Os participantes foram convidados pessoalmente, quando foi apresentada a pesquisa e seus objetivos, solicitando-se a assinatura do Termo de Consentimento Livre e Esclarecido (TCLE). Posteriormente, em horário agendado, foi realizada a coleta de dados, a partir de consulta de enfermagem, e adicionalmente, consultando o prontuário do paciente. Os procedimentos da pesquisa não interferiram na rotina dos serviços de assistência à saúde, conforme Resolução 580/2018 (Brasil, 2018).

Para coleta de dados, foi utilizado um instrumento com variáveis sociodemográficas, como idade, estado civil, renda, entre outros, além de aspectos clínicos dos pacientes como medicações em uso, índice de massa corporal (IMC), complicações durante a hemodiálise, entre outros. Para complementar, foram levantados dados de exames laboratoriais do último mês, hematócrito $(\%)$, hemoglobina $(\mathrm{g} / \mathrm{dl})$, potássio $(\mathrm{mEq} / \mathrm{l})$, uréia pré e pós diálise $(\mathrm{mg} / \mathrm{dl})$, ferro (ug/l), ferritina (ng/ml), Saturação Transferrina A (ng/ml), paratormônio (PTH), Glicose A (mg/dl) e Fósforo (mg/dl).

Os dados foram tabulados em uma planilha do programa Microsoft Excel e, após, sofreram análise estatística por meio do software SPSS (Statistical Package for the Social Sciences), versão 25.0. A apresentação dos resultados ocorreu através da estatística descritiva pelas distribuições absoluta e relativa (n - \%), bem como, pelas medidas de tendência central (média e mediana) e de variabilidade (desvio padrão, amplitude e amplitude interquartílica), com o estudo da simetria das distribuições através do teste de Kolmogorov-Smirnov.

A coleta de dados ocorreu após a anuência formal da instituição coparticipante e aprovação do CEP/Faccat (parecer $n^{\circ}$ 4.260.399 de 4 de setembro de 2020). Todos os participantes consentiram a participação, antes de iniciar o preenchimento do questionário, através do Termo de Consentimento Livre e Esclarecido (TCLE) respeitando os preceitos éticos da resolução 510/2016 (Brasil, 2016).

\section{Resultados}

A população foi composta por 126 pacientes com DRC em tratamento hemodialítico. Houve recusa de vinte e oito pacientes quanto à participação no estudo. Quatro pacientes foram excluídos por óbito ou troca de método dialítico. Assim, a amostra constituiu-se de 94 pacientes.

Os resultados apresentados referem-se a análise descritiva de uma amostra com 94 participantes. Sobre as variáveis demográficas e socioeconômicas, a média de idade foi de 53,1 ( $d p=13,7)$ anos. O sexo masculino alcançou 61,7\% (n=58) dos casos. O estado civil casado(a) ou em relacionamento estável caracterizou 45,7\% (n=43) da amostra, enquanto que, aqueles que se declaram solteiros representaram $27,7 \%(n=26)$.

A maior parte da amostra reside na zona urbana, 78,7\% $(n=74)$. O recebimento de auxílio-doença foi confirmado pela grande maioria dos pacientes, 90,4\% (n=85). O rendimento mensal na faixa de mais de 1 a 2 salários mínimos foi observado em 46,8\% ( $n=8)$ dos casos. Quando questionados sobre quantas pessoas dependiam da renda, 31,9\% ( $\mathrm{n}=30$ ) informaram três pessoas e $30,9 \%(n=29)$ relataram duas pessoas. 
Em relação ao nível de escolaridade, destacou-se o ensino fundamental, 72,4\% (n=68), sendo 62,8\% (n=59) com fundamental incompleto e $9,6 \%(n=9)$ com fundamental completo. A cor branca foi a mais prevalente, 80,9\% ( $n=76)$. A religião católica foi declarada por $69,1 \%(n=65)$. Os dados detalhados são apresentados na Tabela 1.

Tabela 1 - Caracterização demográfica e socioeconômica de pacientes em hemodiálise. Taquara, RS, 2020.

\begin{tabular}{|c|c|c|}
\hline \multirow{2}{*}{ Dados sócio demográficos } & \multicolumn{2}{|c|}{ Amostra (n=94) } \\
\hline & $\mathbf{n}$ & $\%$ \\
\hline \multicolumn{3}{|l|}{ Idade (categorias) } \\
\hline Média \pm desvio padrão & \multicolumn{2}{|c|}{$53,1 \pm 13,7$} \\
\hline Mediana $\left(1^{\circ}-3^{\circ}\right.$ quartil $)$ & \multicolumn{2}{|c|}{$54,0(43,0-63,0)$} \\
\hline \multicolumn{3}{|l|}{ Sexo } \\
\hline Feminino & 36 & 38,3 \\
\hline Masculino & 58 & 61,7 \\
\hline \multicolumn{3}{|l|}{ Estado civil } \\
\hline Casado(a) & 43 & 45,7 \\
\hline Solteiro(a) & 26 & 27,7 \\
\hline Viúvo(a) & 14 & 14,9 \\
\hline Separado(a) & 11 & 11,7 \\
\hline \multicolumn{3}{|l|}{ Tipo de residência } \\
\hline Urbana & 74 & 78,7 \\
\hline Rural & 20 & 21,3 \\
\hline \multicolumn{3}{|l|}{ Recebe auxílio-doença } \\
\hline Não & 9 & 9,6 \\
\hline Sim & 85 & 90,4 \\
\hline \multicolumn{3}{|l|}{ Renda familiar } \\
\hline Até 1 salário mínimo & 27 & 28,7 \\
\hline 1 a 2 salários mínimos & 44 & 46,8 \\
\hline Mais de 2 salários mínimos & 23 & 24,5 \\
\hline \multicolumn{3}{|c|}{ Quantas pessoas dependem da renda } \\
\hline 1 a 2 & 46 & 49,0 \\
\hline 3 a 4 & 43 & 45,7 \\
\hline 5 ou mais & 5 & 5,3 \\
\hline \multicolumn{3}{|l|}{ Escolaridade } \\
\hline Analfabeto & 3 & 3,2 \\
\hline Fundamental incompleto & 59 & 62,8 \\
\hline Fundamental completo & 9 & 9,6 \\
\hline Médio incompleto & 7 & 7,4 \\
\hline Médio completo & 13 & 13,8 \\
\hline Superior completo & 2 & 2,1 \\
\hline Pós-graduação & 1 & 1,1 \\
\hline \multicolumn{3}{|l|}{ Cor autorreferida } \\
\hline Branca & 76 & 80,9 \\
\hline Parda & 18 & 19,1 \\
\hline \multicolumn{3}{|l|}{ Religião } \\
\hline Católica & 65 & 69,1 \\
\hline Evangélica & 17 & 18,1 \\
\hline Outra & 12 & 12,8 \\
\hline
\end{tabular}

Fonte: Dados da pesquisa.

Sobre as causas da DRC, prevaleceram a hipertensão arterial (HAS), 43,6\% (n=41); e Diabetes Mellitus (DM), 20,2\% $(\mathrm{n}=19)$. Ainda, destacou-se a causa "Outros", representando 24,5\% ( $\mathrm{n}=23)$ da amostra, que envolveu, entre outras, as glomerulonefrites e agenesia renal unilateral. No que se refere às informações sobre as características clínicas dos participantes, verificou-se que o tempo de diagnóstico DRC apresentou mediana de 4,0 (1-3 $3^{\circ}$ quartil: 2,0 - 7,0) anos.

O acesso venoso via fístula arteriovenosa para hemodiálise (FAV) foi observado na grande maioria dos casos, $94,7 \%$ (n=89). Em relação à medicação utilizada pelos participantes, as mais frequentes foram Eritropoetina, 67,0\% (n=63); Sacarato de Hidróxido de Ferro, 58,5\% ( $n=55)$, bem como Calcitriol e anti-hipertensivos, com proporções de 54,3\% (n=51) e 53,2\% $(\mathrm{n}=50)$, respectivamente. Quanto ao IMC, a média foi de $25,0(d p=5,1) \mathrm{kg} / \mathrm{m}^{2}$.

No que se refere às complicações ocorridas durante a hemodiálise, destacaram-se as situações de hipotensão 56,4\% $(\mathrm{n}=53)$ e cãimbras, $28,7 \%(\mathrm{n}=27)$. A Tabela 2 apresenta o detalhamento dos dados clínicos. 
Tabela 2 - Caracterização geral dos aspectos clínicos de pacientes em hemodiálise. Taquara, RS, 2020.

\begin{tabular}{|c|c|c|}
\hline \multirow{2}{*}{ Dados clínicos } & \multicolumn{2}{|c|}{$\operatorname{Amostra}(n=94)^{A}$} \\
\hline & $n$ & $\%$ \\
\hline \multicolumn{3}{|l|}{ Causa DRC } \\
\hline DM & 19 & 20,2 \\
\hline HAS & 41 & 43,6 \\
\hline Genéticos & 11 & 11,7 \\
\hline Outros $¥$ & 23 & 24,5 \\
\hline \multicolumn{3}{|l|}{ Tempo de diagnóstico DRC (anos) } \\
\hline Média \pm desvio padrão & \multirow{2}{*}{\multicolumn{2}{|c|}{$\begin{array}{c}6,05 \pm 6,46 \\
4,00(2,00-7,00)\end{array}$}} \\
\hline Mediana $\left(1^{\circ}-3^{\circ}\right.$ quartil $)$ & & \\
\hline \multicolumn{3}{|l|}{ Acesso venoso } \\
\hline FAV & 89 & 94,7 \\
\hline CAT & 5 & 5,3 \\
\hline \multicolumn{3}{|l|}{ Medicações } \\
\hline Calcitriol & 51 & 54,3 \\
\hline Cloridrato de Sevelamer & 25 & 26,6 \\
\hline Eritropoetina & 63 & 67,0 \\
\hline Noripurum & 55 & 58,5 \\
\hline Anti-hipertensivos & 50 & 53,2 \\
\hline Anti-glicêmicos* & 19 & 20,2 \\
\hline \multicolumn{3}{|l|}{$\operatorname{IMC}\left(\mathrm{kg} / \mathrm{m}^{2}\right)$} \\
\hline Média \pm desvio padrão & $25,0 \pm 5,1$ & \\
\hline \multicolumn{3}{|l|}{ Complicações durante a hemodiálise } \\
\hline Mal-estar & 9 & 9,6 \\
\hline Cãimbras & 27 & 28,7 \\
\hline Hipotensão & 53 & 56,4 \\
\hline Hipertensão & 9 & 9,6 \\
\hline Hipoglicemia & 1 & 1,1 \\
\hline Náusea/vômito & 7 & 7,4 \\
\hline Tremores & 1 & 1,1 \\
\hline Fraqueza & 19 & 20,2 \\
\hline
\end{tabular}

A: Percentuais obtidos com base no total da amostra. B: variável com distribuição assimétrica (Kolmogorov Smirnov; p<005). ¥ Outras causas: Glomerulonefrites, agenesia renal unilateral. DM - Diabetes Mellitus. HAS - Hipertensão Arterial Sistêmica. FAV - Fístula Arteriovenosa. CAT - Cateter. *Insulina e/ou anti-glicêmico oral. Fonte: Dados da pesquisa.

No que se refere às informações dos exames laboratoriais, as taxas de hematócrito variaram de 17 a $45 \%$, com média de 29,68 $(d p=5,16) \%$. Para a hemoglobina, os níveis de mínimo de máximo foram de 5 e $15 \mathrm{~g} / \mathrm{dl}$, com média de $9,67(d p=$ $1,73) \mathrm{g} / \mathrm{dl}$. Em relação ao potássio a média foi de $4,83(d p=0,74) \mathrm{mEq} / \mathrm{l}$, sendo que os níveis da amostra oscilaram de 3,00 a 7,00 mEq/l. Cabe salientar que 55,3\% (n=52) dos pacientes apresentaram nível de potássio de 5,00 mEq/l.

Para a uréia, observou-se que na avaliação pré-diálise os níveis variaram de 30 a $239 \mathrm{mg} / \mathrm{dl}$, com média de 137,36 ( $d p$ $=40,03)$ e mediana de 139,50. Quanto à avaliação pós-diálise, a média foi de 43,00 $(d p=22,48) \mathrm{mg} / \mathrm{dl}$ e mediana de 38,00 $\mathrm{mg} / \mathrm{dl}$, sendo que os níveis observados na amostra variaram de 5 a $144 \mathrm{mg} / \mathrm{dl}$. Na comparação dos níveis de uréia entre as duas avaliações (pré e pós) foi detectada diferença estatisticamente significativas [t-Student pareado; $t_{\mathrm{g} .1 .}=93 ; \mathrm{p}<0,001$ ], indicando que, os níveis médios de uréia na avaliação pós se mostraram inferiores aos da avaliação pré.

Sobre os níveis de ferro, a amostra apresentou mediana de 66,00 (1 ${ }^{\circ}-3^{\circ}$ quartil: 45,00 - 90,25) ug/l sendo que as medidas de mínimo e máximo detectadas na amostra foram de 17 e 196 ug/l. Para a ferritina, os níveis observados oscilaram de 1 a 986 ng/ml, com mediana de 189,00 ( $1^{\circ}-3^{\circ}$ quartil: 33,50 - 433,00) ng/ml. Quanto à saturação de transferrina, a mediana foi estimada em 27,00 ( $1^{\circ}-3^{\circ}$ quartil: 17,00 - 37,25) ng/ml. No PTH, foi detectada uma grande amplitude de variação, com o mínimo de 2 e o máximo de $2202 \mathrm{pg} / \mathrm{ml}$; a mediana foi de 173,50 (1 $1^{\circ}-3^{\circ}$ quartil: 78,50 - 438,50) pg/ml. Para a glicose, a mediana foi de 150,50 ( $1^{\circ}-3^{\circ}$ quartil: 105,50 - 237,50) mg/dl, sendo o mínimo de $98 \mathrm{mg} / \mathrm{dl}$ e o máximo de $515 \mathrm{mg} / \mathrm{dl}$. Sobre os níveis de fósforo, estes 
variaram de 1 a $9 \mathrm{mg} / \mathrm{dl}$, com média 4,66 $(d p=1,65) \mathrm{mg} / \mathrm{dl}$. O detalhamento dos exames laboratoriais é apresentado na Tabela 3.

Tabela 3: Caracterização geral dos exames laboratoriais de pacientes em hemodiálise. Taquara, RS, 2020.

\begin{tabular}{|c|c|}
\hline Exames & Amostra $(n=94)$ \\
\hline \multicolumn{2}{|l|}{ Hematócrito (\%) } \\
\hline Média \pm desvio padrão & $29,68 \pm 5,16$ \\
\hline \multicolumn{2}{|l|}{ Hemoglobina (g/dl) } \\
\hline Média \pm desvio padrão & $9,67 \pm 1,73$ \\
\hline \multicolumn{2}{|l|}{ Potássio (mEq/l) } \\
\hline Média \pm desvio padrão & $4,83 \pm 0,74$ \\
\hline \multicolumn{2}{|l|}{ Uréia pré (mg/dl) } \\
\hline Média \pm desvio padrão & $137,36 \pm 40,03$ \\
\hline \multicolumn{2}{|l|}{ Uréia pós (mg/dl) } \\
\hline Média \pm desvio padrão & $43,00 \pm 22,48$ \\
\hline \multicolumn{2}{|l|}{ Ferro (ug/l) } \\
\hline Média \pm desvio padrão & $71,12 \pm 35,24$ \\
\hline \multicolumn{2}{|l|}{ Ferritina $(\mathrm{ng} / \mathrm{ml})$} \\
\hline Média \pm desvio padrão & $265,79 \pm 255,06$ \\
\hline Mediana $\left(1^{\circ}-3^{\circ}\right.$ quartil $)$ & $189,00(35,50-433,00)$ \\
\hline \multicolumn{2}{|l|}{ Sat Transferrina (ng/ml) } \\
\hline Média \pm desvio padrão & $28,69 \pm 14,51$ \\
\hline \multicolumn{2}{|l|}{ PTH } \\
\hline Média \pm desvio padrão & $302,26 \pm 241,45$ \\
\hline Mediana $\left(1^{\circ}-3^{\circ}\right.$ quartil $)$ & $173,50(78,50-438,50)$ \\
\hline \multicolumn{2}{|l|}{ Glicose (mg/dl) } \\
\hline Média \pm desvio padrão & $198,50 \pm 121,43$ \\
\hline Mediana $\left(1^{\circ}-3^{\circ}\right.$ quartil $)$ & $150,50(105,50-237,50)$ \\
\hline \multicolumn{2}{|l|}{ Fósforo $(\mathrm{mg} / \mathrm{dl})$} \\
\hline Média \pm desvio padrão & $4,66 \pm 1,65$ \\
\hline
\end{tabular}

PHT - paratormônio. Fonte: Dados da pesquisa.

\section{Discussão}

Ao realizar um censo junto aos portadores de DRC submetidos ao tratamento hemodialítico, o presente estudo indentificou que a variável idade foi inferior a encontradodo no Censo Brasileiro de Diálise (superior a 65 anos - 41,5\%), enquanto a variável sexo foi semelhantes, prevalecendo o sexo masculino em 58\% (Neves et al., 2020). Outros estudos também identificaram prevalência do sexo masculino (Sousa et al, 2020; Santos, et. al., 2018), o que também é observado em outros países, como naqueles integrantes do Reino Unido (UKRR, 2021).

Em relação às variáveis estado civil, local de residência, se recebem auxílio-doença, renda familiar e número de pessoas dependentes dessa renda, houve semelhança com um estudo realizado com portadores de DRC em 2017, no qual prevaleceu o estado civil casado $(55,6 \%)$ e recebendo auxílio-doença (>35,8\%) (Spigolon et al., 2018). Em estudo realizado em 2018, identificou-se semelhança com a pesquisa em questão, em que 46,4\% da amostra recebia de um (1) a três (3) salários mínimos, já os indivíduos que dependiam dessa renda eram entre duas (2) a quatro (4) pessoas e 184 participantes da amostra residiam no meio urbano (Piccin et. al., 2018).

Quanto à escolaridade, prevaleceu o ensino fundamental incompleto, assim como Spigolon et al. (2018) (54,3\%). Com a baixa escolaridade encontrada na amostra deste estudo, entende-se que os participantes com menor escolaridade podem possuir um menor entendimento sobre seu tratamento. No mesmo sentido, um estudo sobre letramento em saúde, realizado em 2019 com indivíduos submetidos ao tratamento dialítico, identificou que os pacientes possuem dificuldade na compreensão do seu regime de tratamento, e 80,9\% apresentaram letramento em saúde inadequado (Bezerra et al., 2019). Por outro lado, um estudo que visou identificar o impacto de um suporte de autogerenciamento da DRC, utilizando orientações com linguagem compatível 
culturalmente, concluiu que o letramento em saúde não foi uniformemente associado com o autocuidado para o manejo da DRC (Wong et al., 2018).

Sobre as variáveis cor autorreferida e religião, no último Censo Brasileiro, que ocorreu em 2010, 47,73\% autorreferiram ser de raça branca e 64,6\% da população se diz católico (IBGE, 2010), corroborando os dados de nosso trabalho.

Sobre a etiologia da DRC, no presente estudo, se observou que a HAS, foi a maior causadora da perda da função renal, diferente do encontrado em um estudo realizado uma cidade de Minas Gerais, cuja principal etiologia ficou por conta do Diabetes mellitus (DM) (Campos et al., 2020). Um estudo com portadores de DRC apontou que, em 38,4\% de sua amostra, a HAS é causadora da perda da função renal e que a HAS associada com o DM foi encontrada em 33,8\% da amostra (Spigolon et al., 2018). Já nos Estados Unidos, o DM é responsável por 39\% dos casos de DRC e a HAS por 26\% (CDC, 2021). No Reino Unido o DM figura como etiologia de 27,8\% dos casos e a glomerulonefrite responde por 13,7\%. Em nosso estudo obteve-se um resultado elevado, também, para as Glomerulonefrites e Agenesia Renal Unilateral.

Sobre o tempo de diagnóstico, acesso venoso, as medicações utilizadas e IMC, alguns estudos corroboram com essas variáveis, como um estudo realizado junto a idosos com DRC, em que a estimativa de tempo em anos de hemodiálise foi de 2 a 25 anos (Debone et al., 2017). O Censo de Diálise de 2009 a 2018, no que se refere ao acesso para diálise, o uso do cateter de longa permanência dobrou no ano de 2013 em relação aos outros anos (Neves et al., 2020); já em nosso estudo, a grande maioria dos participantes tem a fístula arteriovenosa como acesso permanente. Ainda em estudo realizado por SILVA et. al. (2017) apontou que $84,28 \%$ dos participantes possuíam a fístula arteriovenosa. O censo também apontou que as medicações mais utilizadas são eritropoetina, ferro e calcitriol (Neves et al., 2020), o que é semelhante a este trabalho. O censo não menciona o uso de anti-hipertensivos, medicação que também apresentou uso expressivo junto aos participantes deste estudo.

Diferente dos resultados do presente estudo, uma pesquisa que avaliou o estado nutricional de pacientes em hemodiálise, identificou a maioria dos participantes em eutrofia, com média de IMC 24,4 kg/m2 (Bousquet-Santos et al., 2019).

Um estudo realizado na região do nordeste com 300 pacientes em hemodiálise apontou as câimbras e a hipotensão sucessivamente como as complicações mais frequentes assim como neste estudo, reforçam ainda que essas configuram-se como importantes fatores a serem observados e prevenidos (Tinôco, et. al., 2017).

Sobre os exames laboratoriais, o Censo Brasileiro de Diálise fez comparações em relação às referências internacionais, determinadas pelo KDIGO (Kidney Disease Improving Global Outcomes), e identificou que, no ano de 2018, 29\% dos pacientes tinham hemoglobina $<10 \mathrm{mg} / \mathrm{dl}, 32 \%$ com fósforo $>5,5 \mathrm{mg} / \mathrm{dl}, 18 \%$ com PTH > 600 (Neves et al., 2020). A análise dos dados de nosso estudo também evidenciou valores baixos de hemoglobina, mas os valores de fósforo e de PTH estavam dentro dos limites preconizados pelo KDIGO (KDIGO, 2017).

Uma das limitações deste estudo do tipo censo é que houve negativa expressiva de participação na pesquisa, representando 25,4\% da população. Além disso, há reduzida capacidade de generalização, uma vez que o estudo foi unicêntrico.

\section{Conclusão}

Este estudo contribui para identificar o perfil de pacientes com DRC em hemodiálise em um serviço de referência no interior do Rio Grande do Sul, RS, e, por conseguinte, subsidiar a equipe de saúde para a tomada de decisões. A partir dos dados coletados, foi possível identificar a prevalência de homens, casados, com renda de um a dois salários mínimos, baixa escolaridade e cor branca. Quanto aos aspectos clínicos, a principal etiologia foi a hipertensão arterial, tempo de diagnóstico médio maior do que seis anos e acesso venoso por fístula arteriovenosa. As principais complicações foram hipotensão e câimbras.

Em relação ao perfil clínico foi possível identificar que os exames laboratoriais apresentaram as taxas de hematócrito e hemoglobina variáveis, apontando desde anemia até valores considerados normais. Os níveis médios de ureia na avaliação pós foram estatisticamente significativos e se mostraram inferiores aos da avaliação pré-diálise, configurando depuração adequada 
dessa substância durante o processo dialítico. Para o PTH, foi detectada uma grande amplitude de variação, desde a normalidade até níveis condizentes para indicação de paratireoidectomia. Demais resultados também oscilaram na amostra participante, o que indica necessidade de atenção individualizada em saúde.

Para trabalhos futuros, sugere-se a realização de estudos prospectivos, a fim de avaliar tendências e efetuar comparações.

\section{Referências}

Bezerra, J. N. M. et. al. (2019). Letramento em saúde dos indivíduos submetidos à terapia dialítica. Texto e Contexto- Enfermagem, $28,01-13$.

Bousquet-Santos, K. Costa, L. G. \& Andrade, J. M. L. (2019). Estado nutricional de portadores de doença renal crônica em hemodiálise no Sistema Único de Saúde. Revista Ciência e Saúde Coletiva, 24 (3), 1189-1199.

Brasil. (2009). Resolução No 358 do Conselho Federal de Enfermagem. http://www.cofen.gov.br/ resoluo-cofen-3582009_4384.html

Brasil. (2018). Resolução No 580 do Ministério da Saúde/ Conselho Nacional de Saúde. https://www.in.gov.br/materia//asset_publisher/Kujrw0TZC2Mb/content/id/31546309/do1-2018-07-16-resolucao-n-580-de-22-de-marco-de-2018-31546295

Brasil. (2016). Resolução No 510 do Ministério da Saúde/ Conselho Nacional de Saúde. https://www.in.gov.br/materia//asset_publisher/Kujrw0TZC2Mb/content/id/22917581

Campos, A. R. (2020). Perfil clínico e epidemiológico dos pacientes com doença renal crônica submetidos a hemodiálise em São João Del Rei-MG. Brazilian Journal of Development, 6 (12), 97016-97032.

Chen, T. K., Knicely, D. H., \& Grams, M. E. (2019). Diagnóstico e tratamento de doença renal crônica: Uma revisão. Revista JAMA. 322 (13), $1294-1304$.

Debone, M. C., et al. (2017). Diagnósticos de enfermagem em idosos com doença renal crônica em hemodiálise. Revista Brasileira de Enfermagem, 70 (4), 833-839.

Kidney Disease: Improving Global Outcomes (KDIGO) CKD-MBD Update Work Group. KDIGO 2017 Clinical Practice Guideline Update for the Diagnosis, Evaluation, Prevention, and Treatment of Chronic Kidney Disease-Mineral and Bone Disorder (CKD-MBD). Kidney Int Suppl. 7:1-59.

Kramer, A., et. al. (2020). Relatório anual do registro ERA-EDTA 2017: um resumo. Clinical Kidney Journal, 13 (4), $693-709$.

Murdeshwar, H. \& Anjum, F. (2021). Hemodialysis. https://www.ncbi.nlm.nih.gov/books/NBK563296

Neves, P. D. M. de M. et. al. (2021). Inquérito brasileiro de diálise 2019. Brazilian Journal of Nephrology, 43 (2), $217-227$.

Neves, P. D. M. de M. et al. (2020). Censo Brasileiro de Diálise: análise de dados da década 2009-2018. Brazilian Journal of Nephrology, 42 (2), 191-200

Piccin, C., et. al. (2018). Perfil sociodemográfico e clínico de pacientes renais crônicos em hemodiálise. Revista de Enfermagem UFPE on line, 12 (12), 3212-3220

Sampieri, R. H., Callado, C. F., \& Lucio, M. P. B. (2013). Metodologia da pesquisa. (5a ed.), Penso.

Santos, K. K., et. al. (2018). Perfil epidemiológico de pacientes renais crônicos em tratamento. Revista de Enfermagem UFPE on line, 12 (9), $2293-2300$.

Sousa, F. C. A., et al. (2020). Perfil clínico e sociodemográfico de pacientes hemodialisados. Revista Enfermagem Atual, 93 (31), 01 -06.

Spigolon, D. N. et al. (2018). Diagnóstico de enfermagem de portadores de doença renal em hemodiálise: Estudo transversal. Revista Brasileira de Enfermagem, 71 (4), 2014-2020

Tinôco, J. D. S. et al. (2017). Complicações em pacientes renais crônicos submetidos a hemodiálise. Revista Cogitare de Enfermagem, 22 (4), 01 -13.

Thomé, F. S. et al. (2017). Inquérito Brasileiro de Diálise Crônica 2017. Brazilian Journal of Nephrology, 41 (2), $208-214$.

United Kingdom. (2021). UK Renal registry 23rd Annual Report: data to 31/12/2019. UK Kidney Association. https://ukkidney.org/audit-research/annual-report

United States of America. (2021). Chronic kidney disease in the United States, 2021. https://www.cdc.gov/kidneydisease/publications-resources/ckd-nationalfacts.html

United States Renal Data System, National Institutes of Health, National Institute of Diabetes and Digestive and Kidney Diseases. (2019). Annual data report: epidemiology of kidney disease in the United States. https://www.usrds.org/annual-data-report/

Vallianou, N. G. et. al. (2019). Doença renal crônica e doença cardiovascular: existe alguma relação? Revista Current Cardiology Reviews, 15 (1), $55-63$.

Zheng, Y., Cheung, K. S. L., \& Yip, P. (2020). Estamos vivendo mais e mais saudáveis? Journal of aging and health, 32 (10), $1645-1658$.

Wong, K. K., et. al. (2018). Associação entre alfabetização em saúde e comportamentos de autocuidado entre pacientes com doença renal crônica. BMC Nephrology, 19 (196), 01-08. 